\title{
Ji-hyun Philippa Kim, Pour une littérature médiévale moderne. Gaston Paris, l'amour courtois et les enjeux de la modernité
}

\section{Walter Meliga}

\section{(2) OpenEdition}

\section{Journals}

\section{Edizione digitale}

URL: http://journals.openedition.org/studifrancesi/1223

DOI: $10.4000 /$ studifrancesi. 1223

ISSN: 2421-5856

\section{Editore}

Rosenberg \& Sellier

\section{Edizione cartacea}

Data di pubblicazione: 1 décembre 2015

Paginazione: 558-559

ISSN: 0039-2944

\section{Notizia bibliografica digitale}

Walter Meliga, «Ji-hyun Philippa Kim, Pour une littérature médiévale moderne. Gaston Paris, l'amour courtois et les enjeux de la modernité», Studi Francesi [Online], 177 (LIX | III) | 2015, online dal 01 décembre 2015, consultato il 06 janvier 2021. URL: http://journals.openedition.org/studifrancesi/1223 ; DOI: https://doi.org/10.4000/studifrancesi.1223

Questo documento è stato generato automaticamente il 6 janvier 2021 .

\section{cc) $(9)$}

Studi Francesi è distribuita con Licenza Creative Commons Attribuzione - Non commerciale - Non opere derivate 4.0 Internazionale. 


\title{
Ji-hyun Philippa Kim, Pour une littérature médiévale moderne. Gaston Paris, l'amour courtois et les enjeux de la modernité
}

\author{
Walter Meliga
}

\section{NOTIZIA}

JI-HYUN PHILIPPA KIM, Pour une littérature médiévale moderne. Gaston Paris, l'amour courtois et les enjeux de la modernité, Paris, Champion, 2012 («Essais sur le Moyen Âge», 55), pp. 218.

1 Il lavoro ha come obiettivo lo studio della genesi del celeberrimo concetto di «amour courtois» attraverso l'analisi dei rapporti che il suo inventore Gaston Paris ha intrattenuto con il decadentismo-in relazione sia ai suoi studi sia alle sue frequentazioni personali - e delle ricerche che egli ha dedicato alle opere del Medioevo e a quelle di alcuni artisti del suo tempo. L'amour courtois appare così condensare in sé vari punti di tensione fra Medioevo e modernità e fra adesione sentimentale e ricerca nei confronti del passato: Paris è infatti al centro del processo che in Francia fa della letteratura medievale un oggetto di attività scientifica e dell'indagine storica e filologica su di essa una parte di un sistema di conoscenze analogo a quello delle scienze naturali, in ciò ben distinto dal gusto e dal sentimento romantici di cui fu eminente rappresentante suo padre Paulin.

2 Nel primo capitolo (pp. 17-47), l'autrice analizza le condizioni culturali della Francia della seconda metà del xix secolo in relazione alla situazione e al movimento d'opinione che prende il nome di décadence (dunque non soltanto il Decadentismo letterario), nella quale il nuovo studio del passato rappresentato dalla filologia romanza costituisce un progresso scientifico moderno, unito alla tensione verso il superamento delle condizioni di "decadenza" nella quale era ritenuta versare la Francia stessa del periodo 
intorno alla guerra franco-prussiana. Gaston Paris d'altra parte partecipava alla vita culturale del suo tempo: come si vede nel secondo capitolo (pp. 49-95), egli aveva l'abitudine di tenere dei salons ai quali intervenivano ora i colleghi romanisti ora gli intellettuali e i letterati del momento, fra i quali Paul Bourget - poeta, romanziere e critico letterario, particolarmente interessato alla crisi della décadence nonché alla psicologia amorosa - e Sully Prudhomme - poeta interessato all'amore e alla filosofia. Il terzo capitolo (pp. 97-127) mostra come il concetto di amour courtois - divenuto dopo la sua «invenzione» da parte di Paris nel celebre articolo di «Romania» (1883) una categoria, apprezzata o criticata e tuttavia ineliminabile, degli studi di letteratura medievale - fuoriesce da un lavoro critico sui romanzi della Tavola Rotonda e sul Lancelot in particolare, dove il nuovo concetto serve come criterio di confronto dei romanzi arturiani francesi verso la materia bretone preesistente e dove l'argomento centrale è un altro (i rapporti fra il romanzo di Chrétien e la versione in prosa). L'amour courtois è per Paris un'arte e una scienza insieme, prodotto delle modificazioni che sono state apportate dalla tradizione francese alla leggenda di una regina rapita e poi liberata (Ginevra), e in questo si oppone all'amore tristaniano, rimasto allo stadio "semplice" e "naturale" delle fonti celtiche, salvo poi subire la stessa evoluzione una volta entrato nell'ambiente letterario francese. Dallo studio dell'evoluzione dei miti amorosi di Lancillotto e di Tristano secondo Paris si rileverebbe così «un préjugé» (p. 118) o «une certaine idéologie» (p. 122), per i quali il mito e il suo significato sono "puri" alle origini per divenire oggetto dell'arte e della convenzione nel corso della loro rielaborazione. Il concetto di amour courtois troverebbe così un rapporto con la décadence della cultura letteraria della Francia fin de siècle (già illustrata nel primo capitolo) e anche un certo parallelismo con la stessa pratica di editore critico di Paris, anche se qui (p. 124) il collegamento con la critica del testo di matrice lachmanniana ancorché percorribile nell'idea di una comune visione "degenerativa" della trasmissione-andrebbe sostanziato meglio e più ampiamente che con una sola citazione dal libro, "ideologicamente" antilachmanniano e tecnicamente superficiale, di Bernard Cerquiglini (Éloge de la variante, 1989). Infine, nel quarto capitolo (pp. 129-161) sono discussi gli scritti a proposito di tre personaggi contemporanei - Jules Michelet, Richard Wagner e Frédéric Mistral - pubblicati da Paris in diversi momenti della sua vita e che permettono di approfondire e di sostanziare con nuovi argomenti le ragioni dell'opposizione fra l'amore tristaniano e l'amour courtois. Di Michelet, Paris esamina sfavorevolmente il trattato L'Amour (1858), dove si ritrovano considerazioni sull'amore come "arte" in un periodo di "decadenza" che non possono non richiamare quelle di Paris sull'amour courtois, anche se andrebbe segnalata la discordanza temporale fra queste ultime e l'articolo su Michelet, che precede di più di un ventennio. Di Wagner, l'ultimo narratore di Tristano, che crede ingenuamente all'origine germanica del mito, Paris, in lavori degli anni maturi dal 1883 al 1897, apprezza la capacità di estrarne il sentimento profondo e di riattivarne la forza nel "decadente" mondo moderno. Infine, Mistral, nel 1896 viene apprezzato da Paris per la sua opera linguistica ma forse ancora di più per la sua poesia, che, per quanto difettosa nelle sue connessioni con la letteratura occitana medievale, rappresenta un tentativo di riscatto dalla "decadenza" in cui versava il mondo meridionale e dalla décadence tutta di quel periodo. Come l'autrice osserva nella conclusione (pp. 163-165), il concetto di amour courtois, oggi «incompréhensible» (p. 165), si decodifica attraverso la rilettura insieme della cultura medievale e di quella dell'epoca e dello studioso della sua invenzione, anche se andrebbe ricordato il fatto che le difficoltà che l'amour courtois produce nella ricerca 
odierna sulla letteratura medievale non provengono dalla sua genesi in Paris e nelle condizioni culturali del suo tempo ma piuttosto dall'uso troppo ampio e generico che ne è stato fatto.

3 Chiudono il volume delle Annexes di documenti concernenti Paris (lettere, una conferenza, l'elenco dei corsi al Collège de France), un indice dei nomi e una bibliografia delle fonti primarie e secondarie. 